\title{
Diversity of seed mineral composition of Phaseolus vulgaris L. germplasm
}

\author{
Carla Pinheiro $^{a}$, José P. Baeta ${ }^{b}$, Ana M. Pereira ${ }^{c}$, Hermínia Domingues ${ }^{c}$, Cândido P. Ricardo ${ }^{a, d, *}$ \\ a Plant Biochemistry, Instituto de Tecnologia Química e Biológica, Apartado 127, 2781-901 Oeiras, Portugal \\ ${ }^{\mathrm{b}}$ Estação Florestal Nacional, Quinta do Marquês, 2780-159 Oeiras, Portugal

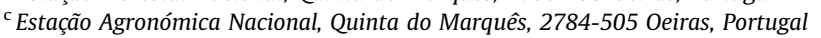 \\ ${ }^{\mathrm{d}}$ Instituto Superior de Agronomia, Tapada da Ajuda, 1349-017 Lisboa, Portugal
}

\section{A R T I C L E I N F O}

\section{Article history:}

Received 29 August 2008

Received in revised form 14 January 2010

Accepted 29 January 2010

\section{Keywords:}

Common bean

Germplasm diversity

Mineral content

Mineral correlations

Phaseolus vulgaris

Seeds

Biodiversity

Cultivar differences and nutrient content

Ancient botanical variety

Food analysis

Food composition

\begin{abstract}
A B S T R A C T
A collection of 155 accessions of ancient Portuguese common beans (Phaseolus vulgaris L.) was evaluated in relation to the content of 8 minerals ( $\mathrm{Zn}, \mathrm{Cu}, \mathrm{Fe}, \mathrm{Mn}, \mathrm{Ca}, \mathrm{Mg}, \mathrm{P}$ and $\mathrm{K}$ ) important for human nutrition. A high degree of variability for $\mathrm{P}, \mathrm{Fe}, \mathrm{Zn}, \mathrm{Cu}, \mathrm{Mn}$ and $\mathrm{Ca}$ was observed in the collection. Total correlation matrix analysis revealed the existence of two important sets of strong positive correlations $(P \leq 0.0001)$, one involving $\mathrm{P}, \mathrm{Fe}, \mathrm{Zn}, \mathrm{Cu}$ and protein, and the other $\mathrm{Ca}$ and $\mathrm{Mn}$. The principal component analysis showed that $\mathrm{Zn}$, Fe and $\mathrm{Cu}$ are highly correlated to the first component (27\% of variability) and $\mathrm{Mn}$ and $\mathrm{Ca}$ to the second component ( $22 \%$ of the variability). The high mineral variability observed in the seeds of this common bean collection could be useful for the selection of cultivars with higher nutrition value and for the improvement of seed nutrition quality traits.
\end{abstract}

(C) 2010 Elsevier Inc. All rights reserved.

\section{Introduction}

Legume seeds are an important staple food and source of dietary minerals that potentially provide all of the 15 essential minerals required by humans (Welch et al., 2000). The common bean (Phaseolus vulgaris L.) is the most important grain legume for direct human consumption and is an extremely diverse crop in terms of morphological variability, uses and cultivation (Broughton et al., 2003). At average levels of usual consumption by people of reduced economic means (15-20 $\mathrm{kg} \mathrm{yr}^{-1}$ ), beans can provide $10-20 \%$ of the adult requirement for a number of nutrients, namely iron, phosphorus, magnesium, manganese, and in lesser degree, zinc, copper and calcium (Broughton et al., 2003). However, the concentrations of Fe, $\mathrm{Zn}$, and $\mathrm{Ca}$ are low when compared to animal food products (Wang et al., 2003). Therefore, increasing the content of those minerals in plant food through breeding is considered a suitable strategy to combat mineral deficiency in human populations (Moraghan and

\footnotetext{
* Corresponding author at: ITQB, Apartado 127, 2781-901 Oeiras, Portugal. Fax: +351214433644.

E-mail address: ricardo@itqb.unl.pt (C.P. Ricardo).
}

Grafton, 2001). Searching for high mineral content cultivars is thus fundamental.

The Iberian Peninsula was an important region of introduction of the American P. vulgaris in Europe, becoming a secondary centre of diversity (Santalla et al., 2002). P. vulgaris rapidly conquered all of Portugal, partially replacing Vigna and the Asian Phaseolus species. As agriculture and society have evolved together, the current state of farming systems is the result of the interaction of climatic, edaphic, biotic and social factors (Broughton et al., 2003); consequently, crop seed composition has been modulated by genotype and environmental interactions. Due to its cleistogamic nature and the diversity of edafic climatic regions in Portugal, a natural selection and genetic drift of $P$. vulgaris led to the appearance of numerous local forms still in cultivation. Since these local forms were grown in the same soil for centuries they are somehow the result of the farmer's selection, who, year after year, chose varieties of high production.

This observation implies that $P$. vulgaris forms originating from very different soil types (reflecting the geological substrates) may have quite distinct genetic characteristics in relation to mineral uptake and use efficiency. This fact together with the suggestion that $P$. vulgaris seeds are a good nutritional source of several minerals prompted us to evaluate the mineral composition of a collection of local accessions from all over the country. 


\section{Material and methods}

\subsection{Plant sampling}

The 155 accessions studied in this work belong to the $P$. vulgaris germplasm collection stored at the EAN Germplasm Bank (Oeiras, Portugal), and were originated from the regions shown in Fig. 1.

All the seeds assayed resulted from plants grown at Oeiras (Quinta do Marquês) in the same Anthrosol (WRB, 2006) fertilised at sowing with $300 \mathrm{~kg} / \mathrm{ha} 1: 3: 3(\mathrm{~N}: \mathrm{P}: \mathrm{K})$ and with the characteristics shown in Table 1 . The main soil characteristics were determined through the methods adopted by the Soil Conservation Service (1972): Soil $\mathrm{PO}_{4}$ and $\mathrm{K}_{2} \mathrm{O}$ availability was determined by the Egner-Riehm method (Egner et al., 1960), and $\mathrm{Cu}, \mathrm{Zn}, \mathrm{Fe}$ and $\mathrm{Mn}$ were extracted by the diethylenetriaminepentaacetic acid (DTPA) method (Lindsay and Norvell, 1978), and analysed by atomic absorption spectrometry as described below for the seeds.

From each accession, 20 plants were grown in a row and care was taken to check that each plant pertained to that specific accession. At harvest, each plant was collected individually and the seeds of 3 of its representative pods were gathered. A biological sample refers to 3 seeds from an individual plant.

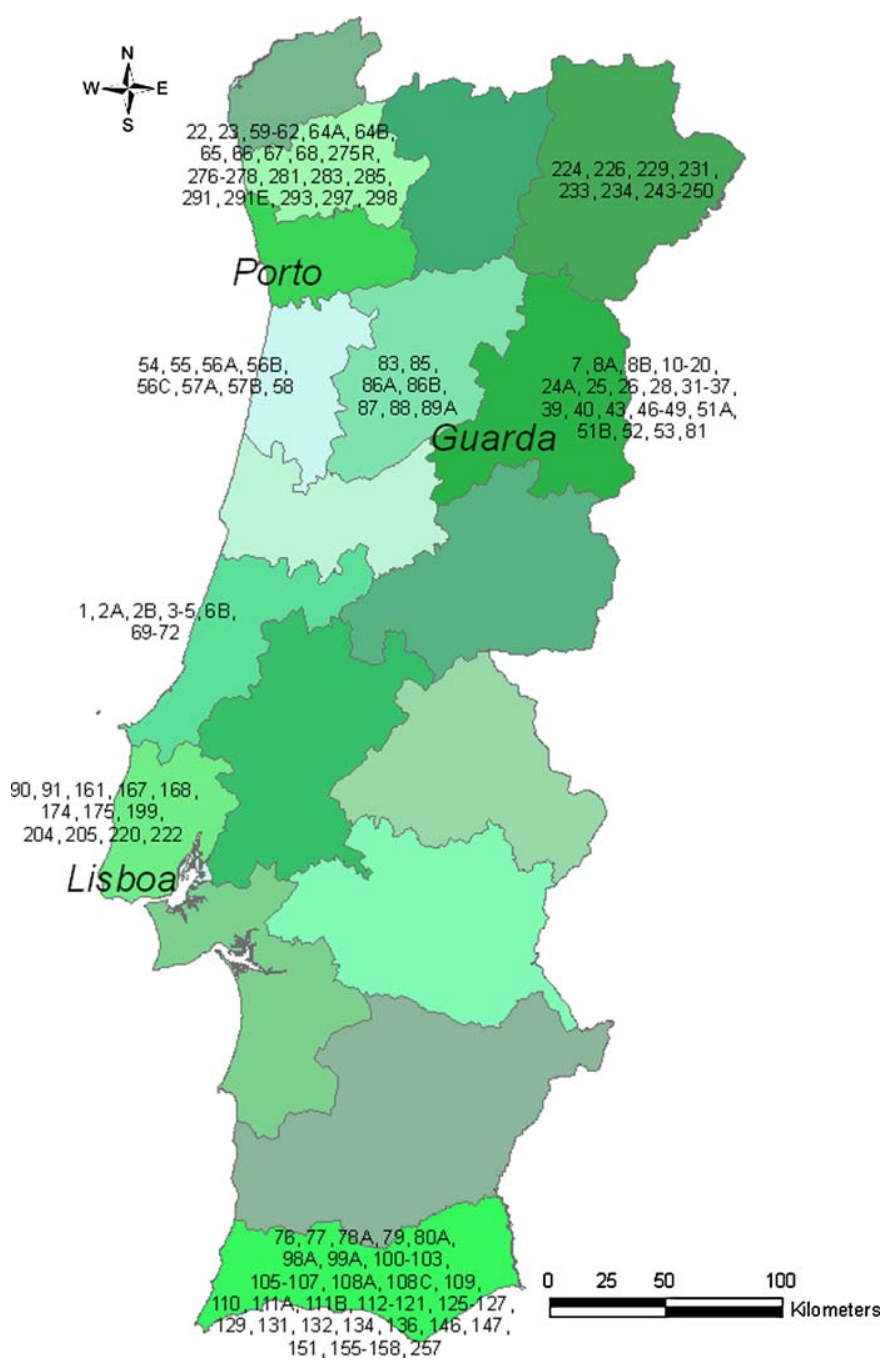

Fig. 1. Map of Portugal showing the collection sites of the several P. vulgaris

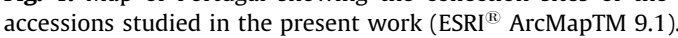

Table 1

Physical characteristics and chemical data (0-30 cm depth layer) from the Anthrosol (WRB, 2006) where the 155 P. vulgaris accessions were grown.

\begin{tabular}{lc}
\hline Coarse sand (\%) & 12.8 \\
Fine sand (\%) & 29.7 \\
$\mathrm{Silt}(\%)$ & 24.5 \\
$\mathrm{Clay} \mathrm{( \% )}$ & 33.0 \\
$\mathrm{pH}\left(\mathrm{H}_{2} \mathrm{O}\right)$ & 7.9 \\
$\mathrm{CaCO}_{3}(\%)$ & 6.0 \\
Organic matter (\%) & 1.51 \\
Organic C (\%) & 0.88 \\
Total N (\%) & 0.14 \\
Available $\mathrm{PO}_{4}\left(\mathrm{mg} \mathrm{kg}^{-1}\right)$ & 422 \\
Available $\mathrm{K}_{2} \mathrm{O}\left(\mathrm{mg} \mathrm{kg}^{-1}\right)$ & 378 \\
Available $\mathrm{Cu}\left(\mathrm{mg} \mathrm{kg}^{-1}\right)$ & 1.35 \\
Available $\mathrm{Fe}\left(\mathrm{mg} \mathrm{kg}^{-1}\right)$ & 3.81 \\
Available $\mathrm{Mn}\left(\mathrm{mg} \mathrm{kg}^{-1}\right)$ & 7.77 \\
Available $\mathrm{Zn}\left(\mathrm{mg} \mathrm{kg}^{-1}\right)$ & 0.87 \\
\hline
\end{tabular}

\subsection{Mineral composition analysis}

For the determination of the seed minerals, $\mathrm{Zn}, \mathrm{P}, \mathrm{Cu}, \mathrm{Fe}, \mathrm{Mg}$, $\mathrm{Mn}$, Ca and K, triplicate biological samples $(n=3)$ from each accession were analysed. The seeds were washed with deionised water, dried at $80{ }^{\circ} \mathrm{C}$, weighed and ashed at $450{ }^{\circ} \mathrm{C}$ in a muffle furnace. The ashes were dissolved in $5 \mathrm{~mL}$ of $20 \%(\mathrm{v} / \mathrm{v}) \mathrm{HCl}$ and diluted to a volume of $100 \mathrm{~mL}$ with deionised water. This solution was analysed for $\mathrm{Cu}, \mathrm{Zn}, \mathrm{Fe}, \mathrm{Mn}, \mathrm{Ca}, \mathrm{Mg}$ and $\mathrm{K}$ using a Perkin-Elmer 5000 flame (air-acetylene) atomic absorption spectrometer with hollow-cathode lamp tubes (Norwalk, Connecticut, USA), according to Chapman and Pratt (1961) and Anon. (1971). Phosphorus was measured in $5 \mathrm{~mL}$ of the same solution by the colorimetric molybdenum ammonium vanadate method (Black et al., 1965), using a Hitachi Perkin-Elmer Model-139 UV-vis spectrophotometer (Tokyo, Japan), at the wavelength of $470 \mathrm{~nm}$.

For the atomic absorption spectrophotometer analyses, the linear ranges $\left(\mathrm{mg} \mathrm{l}^{-1}\right)$ were: $\mathrm{Ca}=7 ; \mathrm{Cu}$ and $\mathrm{Fe}=5 ; \mathrm{Mn}=3 ; \mathrm{K}=2$; $\mathrm{Zn}=1$ and $\mathrm{Mg}=0.5$; the detection limits $\left(\mathrm{mg} \mathrm{l}^{-1}\right)$ were: $\mathrm{Fe}=0.01$; $\mathrm{K}=0.005 ; \mathrm{Mn}, \mathrm{Cu}$, and $\mathrm{Zn}=0.002 ; \mathrm{Ca}=0.001 ; \mathrm{Mg}=0.0001$; the sensitivities for $1 \%$ absorption $\left(\mathrm{mg} \mathrm{l}^{-1}\right)$ were: $\mathrm{Fe}$ and $\mathrm{Cu}=0.1$; $\mathrm{Ca}=0.07 ; \mathrm{Mn}=0.05 ; \mathrm{K} \mathrm{0.02;} \mathrm{Zn}=0.015$ and $\mathrm{Mg}=0.007$. For the UV-vis spectrophotometer $P$ determination, the linear range was $20.0 \mathrm{mg} \mathrm{l}^{-1}$.

\subsection{Protein analysis}

Seed protein content was determined by Palha et al. (1988), and calculated from the total nitrogen measured by the Kjeldhal method.

\subsection{Statistical analysis}

The data were analysed by principal component analysis (PCA), using the correlation matrix, to determine the variables containing the maximum possible variance (first, second and third coordinate axes). The statistical software utilised was the JMP In 5.1 (SAS Institute, Cary, NC, USA).

\section{Results}

Table 2 shows the concentration of $\mathrm{Zn}, \mathrm{Cu}, \mathrm{Fe}, \mathrm{Mg}, \mathrm{Mn}, \mathrm{Ca}, \mathrm{K}$ and $P$ in the seeds of the 155 accessions of $P$. vulgaris representative of the Portuguese cultivation regions shown in Fig. 1. Table 2 also contains the seed protein content, as determined by Palha et al. (1988).

High diversity in the mineral composition was found for the accessions of this germplasm collection. For each of the 8 minerals 
Table 2

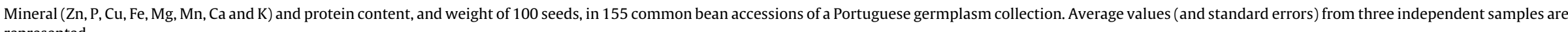
represented.

\begin{tabular}{|c|c|c|c|c|c|c|c|c|c|c|c|}
\hline Accession & $\begin{array}{l}\text { Weight of } \\
100 \text { seeds ( } \mathrm{g} \text { ) }\end{array}$ & $\mathrm{Zn}(\mathrm{ppm})$ & $\mathrm{Cu}(\mathrm{ppm})$ & $\mathrm{Fe}(\mathrm{ppm})$ & $\mathrm{Mn}(\mathrm{ppm})$ & $\mathrm{Ca}(\%)$ & $\mathrm{Mg}(\%)$ & $\mathrm{K}(\%)$ & $\mathrm{P}(\%)$ & $\operatorname{Protein}^{\mathrm{a}}(\%)$ & Region \\
\hline 1 & $70.9 \pm 2.1$ & $36.9 \pm 0.5$ & $11.8 \pm 0.7$ & $65.3 \pm 9.3$ & $12.7 \pm 0.4$ & $0.089 \pm 0.006$ & $0.191 \pm 0.005$ & $1.85 \pm 0.07$ & $0.542 \pm 0.026$ & 24.9 & Leiria \\
\hline $2 \mathrm{~A}$ & $46.2 \pm 0.7$ & $27.5 \pm 0.8$ & $9.5 \pm 0.5$ & $37.5 \pm 3.9$ & $11.9 \pm 0.5$ & $0.102 \pm 0.017$ & $0.208 \pm 0.002$ & $1.97 \pm 0.05$ & $0.465 \pm 0.046$ & 27.6 & Leiria \\
\hline $2 \mathrm{~B}$ & $60.6 \pm 1.9$ & $33.6 \pm 1.9$ & $11.6 \pm 1.4$ & $55.2 \pm 5.4$ & $10.6 \pm 0.6$ & $0.101 \pm 0.015$ & $0.206 \pm 0.021$ & $1.95 \pm 0.02$ & $0.519 \pm 0.033$ & 25.7 & Leiria \\
\hline 3 & $68.6 \pm 2.4$ & $36.2 \pm 3.8$ & $9.2 \pm 0.6$ & $53.9 \pm 4.8$ & $11.8 \pm 0.7$ & $0.089 \pm 0.015$ & $0.216 \pm 0.005$ & $1.81 \pm 0.10$ & $0.552 \pm 0.058$ & 27.6 & Leiria \\
\hline 4 & $58.3 \pm 1.5$ & $41.4 \pm 1.9$ & $10.5 \pm 0.4$ & $84.6 \pm 3.7$ & $10.6 \pm 0.5$ & $0.112 \pm 0.013$ & $0.220 \pm 0.102$ & $1.84 \pm 0.05$ & $0.489 \pm 0.010$ & 29.9 & Leiria \\
\hline 5 & $57.5 \pm 1.8$ & $29.4 \pm 1.0$ & $8.9 \pm 2.1$ & $45.4 \pm 3.7$ & $8.4 \pm 0.3$ & $0.095 \pm 0.007$ & $0.177 \pm 0.030$ & $1.75 \pm 0.08$ & $0.514 \pm 0.026$ & 27.0 & Leiria \\
\hline $6 B$ & $39.2 \pm 2.5$ & $30.6 \pm 4.0$ & $9.1 \pm 0.8$ & $53.5 \pm 3.4$ & $10.4 \pm 0.4$ & $0.090 \pm 0.023$ & $0.242 \pm 0.020$ & $1.74 \pm 0.10$ & $0.496 \pm 0.038$ & 25.8 & Leiria \\
\hline 7 & $56.3 \pm 1.8$ & $31.6 \pm 2.8$ & $11.5 \pm 0.7$ & $50.8 \pm 5.1$ & $10.1 \pm 0.6$ & $0.137 \pm 0.048$ & $0.226 \pm 0.022$ & $1.73 \pm 0.01$ & $0.528 \pm 0.041$ & 26.2 & Guarda \\
\hline $8 \mathrm{~A}$ & $68.7 \pm 2.8$ & $32.8 \pm 2.3$ & $8.9 \pm 1.1$ & $49.6 \pm 3.0$ & $10.4 \pm 0.6$ & $0.124 \pm 0.008$ & $0.221 \pm 0.012$ & $1.65 \pm 0.11$ & $0.568 \pm 0.045$ & & Guarda \\
\hline $8 \mathrm{~B}$ & $67.0 \pm 2.7$ & $32.0 \pm 1.7$ & $7.5 \pm 0.1$ & $52.9 \pm 3.9$ & $10.3 \pm 0.5$ & $0.151 \pm 0.028$ & $0.210 \pm 0.006$ & $1.64 \pm 0.08$ & $0.498 \pm 0.013$ & & Guarda \\
\hline 10 & $55.4 \pm 0.9$ & $42.8 \pm 4.5$ & $10.5 \pm 0.6$ & $63.8 \pm 1.5$ & $12.2 \pm 0.6$ & $0.125 \pm 0.009$ & $0.192 \pm 0.001$ & $1.64 \pm 0.05$ & $0.557 \pm 0.050$ & 27.5 & Guarda \\
\hline 11 & $41.7 \pm 2.5$ & $29.4 \pm 3.5$ & $8.7 \pm 0.2$ & $39.3 \pm 7.4$ & $10.0 \pm 0.7$ & $0.107 \pm 0.029$ & $0.204 \pm 0.005$ & $1.66 \pm 0.03$ & $0.478 \pm 0.035$ & 25.3 & Guarda \\
\hline 12 & $62.0 \pm 0.9$ & $35.4 \pm 6.5$ & $11.0 \pm 0.6$ & $54.1 \pm 2.9$ & $11.2 \pm 0.6$ & $0.098 \pm 0.006$ & $0.180 \pm 0.001$ & $1.66 \pm 0.13$ & $0.510 \pm 0.025$ & & Guarda \\
\hline 13 & $45.5 \pm 1.2$ & $24.7 \pm 3.7$ & $9.7 \pm 1.2$ & $48.6 \pm 9.0$ & $11.3 \pm 0.3$ & $0.136 \pm 0.018$ & $0.201 \pm 0.009$ & $1.74 \pm 0.06$ & $0.445 \pm 0.045$ & 27.2 & Guarda \\
\hline 14 & $51.8 \pm 0.8$ & $27.2 \pm 0.7$ & $9.5 \pm 0.8$ & $42.1 \pm 4.9$ & $12.9 \pm 1.2$ & $0.152 \pm 0.033$ & $0.186 \pm 0.014$ & $1.61 \pm 0.05$ & $0.493 \pm 0.095$ & 25.3 & Guarda \\
\hline 15 & $47.1 \pm 2.4$ & $31.3 \pm 4.9$ & $8.3 \pm 0.1$ & $52.0 \pm 4.8$ & $10.2 \pm 0.4$ & $0.076 \pm 0.012$ & $0.193 \pm 0.005$ & $1.91 \pm 0.05$ & $0.487 \pm 0.039$ & & Sabugal \\
\hline 16 & $59.8 \pm 0.3$ & $31.6 \pm 0.4$ & $10.5 \pm 0.6$ & $52.4 \pm 2.5$ & $10.6 \pm 0.7$ & $0.112 \pm 0.020$ & $0.202 \pm 0.009$ & $1.83 \pm 0.13$ & $0.511 \pm 0.014$ & 25.0 & Sabugal \\
\hline 17 & $50.2 \pm 3.3$ & $34.4 \pm 2.1$ & $11.1 \pm 0.9$ & $59.9 \pm 3.5$ & $11.1 \pm 0.1$ & $0.137 \pm 0.019$ & $0.217 \pm 0.008$ & $1.77 \pm 0.05$ & $0.519 \pm 0.026$ & 24.0 & Sabugal \\
\hline 18 & $37.5 \pm 2.9$ & $41.4 \pm 4.6$ & $12.6 \pm 0.6$ & $70.2 \pm 8.4$ & $16.2 \pm 1.5$ & $0.199 \pm 0.037$ & $0.253 \pm 0.026$ & $1.65 \pm 0.05$ & $0.658 \pm 0.061$ & 26.9 & Sabugal \\
\hline 19 & $48.9 \pm 1.0$ & $29.5 \pm 2.4$ & $9.3 \pm 0.6$ & $46.6 \pm 4.4$ & $11.2 \pm 0.8$ & $0.110 \pm 0.005$ & $0.202 \pm 0.003$ & $1.86 \pm 0.07$ & $0.484 \pm 0.016$ & 23.5 & Sabugal \\
\hline 20 & $66.0 \pm 3.1$ & $26.8 \pm 1.8$ & $10.0 \pm 1.0$ & $39.8 \pm 3.0$ & $8.6 \pm 0.7$ & $0.101 \pm 0.015$ & $0.158 \pm 0.006$ & $1.60 \pm 0.08$ & $0.479 \pm 0.041$ & 25.3 & Sabugal \\
\hline 22 & $28.1 \pm 1.4$ & $27.6 \pm 1.0$ & $9.0 \pm 0.3$ & $48.4 \pm 5.2$ & $13.1 \pm 1.1$ & $0.106 \pm 0.010$ & $0.200 \pm 0.008$ & $1.66 \pm 0.03$ & $0.462 \pm 0.034$ & 25.9 & Sabugal \\
\hline 23 & $43.4 \pm 0.5$ & $34.4 \pm 2.9$ & $10.6 \pm 0.7$ & $59.3 \pm 2.3$ & $9.8 \pm 1.2$ & $0.126 \pm 0.021$ & $0.179 \pm 0.006$ & $1.65 \pm 0.06$ & $0.505 \pm 0.028$ & 23.5 & Braga \\
\hline $24 \mathrm{~A}$ & $53.2 \pm 0.9$ & $34.5 \pm 3.2$ & $11.3 \pm 0.6$ & $67.1 \pm 3.5$ & $10.9 \pm 0.7$ & $0.122 \pm 0.022$ & $0.187 \pm 0.010$ & $1.73 \pm 0.01$ & $0.563 \pm 0.038$ & 28.2 & Guarda \\
\hline 25 & $43.4 \pm 1.7$ & $28.9 \pm 2.2$ & $8.8 \pm 0.2$ & $41.1 \pm 4.8$ & $9.8 \pm 0.2$ & $0.125 \pm 0.011$ & $0.200 \pm 0.004$ & $1.67 \pm 0.01$ & $0.492 \pm 0.010$ & 24.6 & Guarda \\
\hline 26 & $67.8 \pm 1.0$ & $33.9 \pm 1.4$ & $12.2 \pm 0.4$ & $52.2 \pm 5.8$ & $10.4 \pm 0.5$ & $0.133 \pm 0.016$ & $0.212 \pm 0.005$ & $1.64 \pm 0.12$ & $0.560 \pm 0.021$ & 27.6 & Guarda \\
\hline 28 & $49.1 \pm 4.0$ & $37.4 \pm 5.2$ & $10.3 \pm 0.8$ & $46.4 \pm 2.9$ & $9.4 \pm 0.4$ & $0.121 \pm 0.014$ & $0.246 \pm 0.031$ & $1.64 \pm 0.11$ & $0.527 \pm 0.019$ & 25.1 & Guarda \\
\hline 31 & $66.5 \pm 3.6$ & $31.9 \pm 2.9$ & $10.8 \pm 0.3$ & $49.0 \pm 7.7$ & $10.5 \pm 0.4$ & $0.122 \pm 0.016$ & $0.224 \pm 0.006$ & $1.56 \pm 0.05$ & $0.501 \pm 0.052$ & & Guarda \\
\hline 32 & $57.3 \pm 0.6$ & $35.4 \pm 1.6$ & $10.0 \pm 0.4$ & $56.7 \pm 6.7$ & $8.7 \pm 0.3$ & $0.088 \pm 0.003$ & $0.196 \pm 0.006$ & $1.59 \pm 0.03$ & $0.528 \pm 0.022$ & 25.5 & Guarda \\
\hline 33 & $46.6 \pm 1.1$ & $41.3 \pm 8.1$ & $11.4 \pm 0.2$ & $57.1 \pm 3.6$ & $10.5 \pm 0.6$ & $0.095 \pm 0.017$ & $0.211 \pm 0.006$ & $1.81 \pm 0.05$ & $0.583 \pm 0.023$ & 28.8 & Guarda \\
\hline 34 & $61.1 \pm 2.1$ & $37.7 \pm 2.6$ & $11.1 \pm 0.3$ & $57.5 \pm 4.9$ & $11.4 \pm 0.8$ & $0.124 \pm 0.018$ & $0.206 \pm 0.006$ & $1.54 \pm 0.08$ & $0.596 \pm 0.004$ & 25.5 & Guarda \\
\hline 35 & $38.7 \pm 1.0$ & $32.6 \pm 2.4$ & $10.2 \pm 0.1$ & $51.0 \pm 1.5$ & $9.9 \pm 0.5$ & $0.109 \pm 0.013$ & $0.176 \pm 0.003$ & $1.59 \pm 0.01$ & $0.510 \pm 0.027$ & 24.0 & Guarda \\
\hline 36 & $32.4 \pm 0.7$ & $38.1 \pm 1.9$ & $12.7 \pm 0.7$ & $56.3 \pm 4.2$ & $10.6 \pm 0.6$ & $0.136 \pm 0.019$ & $0.201 \pm 0.008$ & $2.04 \pm 0.08$ & $0.572 \pm 0.039$ & 26.6 & Guarda \\
\hline 37 & $46.1 \pm 1.1$ & $36.4 \pm 0.9$ & $12.8 \pm 0.6$ & $59.8 \pm 4.7$ & $11.6 \pm 0.6$ & $0.130 \pm 0.017$ & $0.169 \pm 0.005$ & $1.60 \pm 0.03$ & $0.592 \pm 0.022$ & 30.0 & Guarda \\
\hline 39 & $65.9 \pm 4.1$ & $32.1 \pm 2.2$ & $11.2 \pm 0.2$ & $42.6 \pm 2.1$ & $9.8 \pm 0.2$ & $0.097 \pm 0.004$ & $0.155 \pm 0.005$ & $1.34 \pm 0.05$ & $0.528 \pm 0.007$ & 29.7 & Guarda \\
\hline 43 & $56.8 \pm 3.0$ & $36.2 \pm 1.7$ & $11.5 \pm 0.4$ & $54.5 \pm 2.8$ & $11.4 \pm 1.8$ & $0.158 \pm 0.020$ & $0.185 \pm 0.015$ & $1.48 \pm 0.04$ & $0.516 \pm 0.005$ & 27.5 & Guarda \\
\hline 46 & $51.5 \pm 0.3$ & $32.3 \pm 2.1$ & $9.9 \pm 0.5$ & $39.7 \pm 4.3$ & $10.8 \pm 0.6$ & $0.120 \pm 0.006$ & $0.161 \pm 0.001$ & $1.26 \pm 0.02$ & $0.474 \pm 0.013$ & & Guarda \\
\hline 47 & $51.3 \pm 1.6$ & $27.5 \pm 0.6$ & $9.0 \pm 0.7$ & $37.2 \pm 1.8$ & $12.3 \pm 1.5$ & $0.180 \pm 0.017$ & $0.191 \pm 0.004$ & $1.58 \pm 0.05$ & $0.453 \pm 0.002$ & 25.4 & Guarda \\
\hline 48 & $42.6 \pm 0.6$ & $43.7 \pm 10.2$ & $11.7 \pm 1.6$ & $48.7 \pm 6.6$ & $11.1 \pm 0.9$ & $0.105 \pm 0.003$ & $0.178 \pm 0.009$ & $1.55 \pm 0.10$ & $0.629 \pm 0.089$ & 29.3 & Guarda \\
\hline 49 & $33.8 \pm 0.8$ & $37.7 \pm 5.6$ & $10.7 \pm 0.6$ & $51.5 \pm 6.7$ & $11.2 \pm 1.8$ & $0.139 \pm 0.039$ & $0.171 \pm 0.005$ & $1.35 \pm 0.06$ & $0.596 \pm 0.033$ & 25.5 & Guarda \\
\hline $51 \mathrm{~A}$ & $43.2 \pm 1.8$ & $40.1 \pm 1.6$ & $9.6 \pm 0.4$ & $60.3 \pm 4.2$ & $12.6 \pm 1.0$ & $0.135 \pm 0.015$ & $0.175 \pm 0.001$ & $1.60 \pm 0.03$ & $0.498 \pm 0.031$ & 28.7 & Guarda \\
\hline $51 \mathrm{~B}$ & $36.2 \pm 0.9$ & $42.5 \pm 2.2$ & $10.4 \pm 0.3$ & $64.5 \pm 4.6$ & $12.9 \pm 0.3$ & $0.137 \pm 0.002$ & $0.168 \pm 0.015$ & $1.65 \pm 0.07$ & $0.547 \pm 0.009$ & 27.7 & Guarda \\
\hline 52 & $54.8 \pm 0.5$ & $35.7 \pm 1.4$ & $11.0 \pm 0.4$ & $55.5 \pm 3.4$ & $11.0 \pm 0.2$ & $0.121 \pm 0.009$ & $0.152 \pm 0.005$ & $1.72 \pm 0.03$ & $0.557 \pm 0.045$ & & Guarda \\
\hline 54 & $52.5 \pm 0.9$ & $36.5 \pm 1.0$ & $13.5 \pm 1.0$ & $61.7 \pm 2.2$ & $12.8 \pm 0.7$ & $0.087 \pm 0.010$ & $0.172 \pm 0.009$ & $1.63 \pm 0.11$ & $0.537 \pm 0.010$ & 28.5 & Aveiro \\
\hline 55 & $50.8 \pm 2.9$ & $28.2 \pm 1.3$ & $8.4 \pm 0.3$ & $52.7 \pm 3.8$ & $14.1 \pm 0.1$ & $0.188 \pm 0.004$ & $0.156 \pm 0.005$ & $1.49 \pm 0.05$ & $0.461 \pm 0.025$ & 27.8 & Aveiro \\
\hline $56 \mathrm{~A}$ & $58.0 \pm 0.5$ & $42.3 \pm 2.0$ & $10.2 \pm 1.0$ & $50.2 \pm 3.4$ & $12.4 \pm 0.8$ & $0.157 \pm 0.009$ & $0.228 \pm 0.002$ & $1.64 \pm 0.02$ & $0.615 \pm 0.046$ & 26.7 & Aveiro \\
\hline $56 \mathrm{~B}$ & $52.4 \pm 0.6$ & $36.5 \pm 2.0$ & $12.9 \pm 1.8$ & $67.5 \pm 12.3$ & $15.8 \pm 1.4$ & $0.138 \pm 0.012$ & $0.189 \pm 0.010$ & $1.82 \pm 0.05$ & $0.554 \pm 0.030$ & 25.5 & Aveiro \\
\hline $56 C$ & $47.5 \pm 1.6$ & $41.3 \pm 2.3$ & $11.8 \pm 0.5$ & $74.5 \pm 9.7$ & $14.3 \pm 2.0$ & $0.167 \pm 0.035$ & $0.186 \pm 0.010$ & $1.71 \pm 0.06$ & $0.487 \pm 0.046$ & & Aveiro \\
\hline $57 \mathrm{~A}$ & $42.0 \pm 1.2$ & $35.1 \pm 3.0$ & $11.6 \pm 0.2$ & $68.9 \pm 4.1$ & $12.9 \pm 1.3$ & $0.150 \pm 0.010$ & $0.156 \pm 0.001$ & $1.66 \pm 0.11$ & $0.521 \pm 0.018$ & 26.4 & Aveiro \\
\hline 57B & $36.7 \pm 1.6$ & $34.4 \pm 0.2$ & $11.7 \pm 0.6$ & $54.4 \pm 5.1$ & $13.1 \pm 0.5$ & $0.101 \pm 0.022$ & $0.179 \pm 0.008$ & $1.68 \pm 0.10$ & $0.513 \pm 0.034$ & 26.6 & Aveiro \\
\hline 58 & $41.7 \pm 0.7$ & $36.6 \pm 3.2$ & $11.4 \pm 0.7$ & $67.2 \pm 5.6$ & $11.7 \pm 0.6$ & $0.107 \pm 0.015$ & $0.161 \pm 0.003$ & $1.58 \pm 0.04$ & $0.533 \pm 0.038$ & 24.9 & Aveiro \\
\hline 59 & $47.0 \pm 0.6$ & $36.0 \pm 2.2$ & $11.5 \pm 0.1$ & $70.3 \pm 3.8$ & $11.9 \pm 0.2$ & $0.114 \pm 0.012$ & $0.175 \pm 0.002$ & $1.70 \pm 0.03$ & $0.585 \pm 0.016$ & 26.0 & Minho \\
\hline 60 & $58.2 \pm 1.5$ & $41.8 \pm 3.1$ & $12.2 \pm 0.3$ & $45.8 \pm 1.8$ & $9.8 \pm 0.7$ & $0.082 \pm 0.002$ & $0.150 \pm 0.004$ & $1.69 \pm 0.06$ & $0.576 \pm 0.053$ & 28.7 & Minho \\
\hline 61 & $59.4 \pm 1.0$ & $35.4 \pm 2.7$ & $8.8 \pm 0.3$ & $62.6 \pm 6.4$ & $14.7 \pm 1.8$ & $0.200 \pm 0.010$ & $0.166 \pm 0.006$ & $1.57 \pm 0.05$ & $0.424 \pm 0.015$ & 25.4 & Minho \\
\hline
\end{tabular}


Table 2 (Continued)

\begin{tabular}{|c|c|c|c|c|c|c|c|c|c|c|c|}
\hline Accession & $\begin{array}{l}\text { Weight of } \\
100 \text { seeds (g) }\end{array}$ & Zn (ppm) & $\mathrm{Cu}(\mathrm{ppm})$ & $\mathrm{Fe}(\mathrm{ppm})$ & $\mathrm{Mn}(\mathrm{ppm})$ & Ca (\%) & $\operatorname{Mg}(\%)$ & K (\%) & P (\%) & $\operatorname{Protein}^{\mathrm{a}}(\%)$ & Region \\
\hline 62 & $24.9 \pm 0.3$ & $37.5 \pm 1.0$ & $9.9 \pm 2.0$ & $77.6 \pm 6.1$ & $16.0 \pm 0.9$ & $0.158 \pm 0.007$ & $0.217 \pm 0.017$ & $1.48 \pm 0.03$ & $0.545 \pm 0.015$ & 23.6 & Minho \\
\hline $64 \mathrm{~A}$ & $40.5 \pm 0.5$ & $33.5 \pm 1.6$ & $8.7 \pm 1.0$ & $68.6 \pm 2.7$ & $13.2 \pm 0.4$ & $0.195 \pm 0.027$ & $0.208 \pm 0.005$ & $1.47 \pm 0.06$ & $0.513 \pm 0.043$ & 24.4 & Minho \\
\hline $64 \mathrm{~B}$ & $42.0 \pm 1.3$ & $37.6 \pm 2.5$ & $10.8 \pm 0.2$ & $75.9 \pm 3.5$ & $15.7 \pm 2.4$ & $0.151 \pm 0.033$ & $0.187 \pm 0.008$ & $1.50 \pm 0.03$ & $0.544 \pm 0.029$ & & Minho \\
\hline 65 & $54.6 \pm 0.2$ & $32.0 \pm 0.9$ & $9.7 \pm 0.6$ & $54.5 \pm 1.6$ & $12.5 \pm 0.4$ & $0.123 \pm 0.013$ & $0.185 \pm 0.001$ & $1.41 \pm 0.08$ & $0.466 \pm 0.025$ & 26.0 & Minho \\
\hline $66 \mathrm{~A}$ & $47.3 \pm 1.2$ & $34.4 \pm 1.4$ & $11.9 \pm 0.4$ & $56.3 \pm 4.0$ & $10.2 \pm 0.7$ & $0.094 \pm 0.020$ & $0.202 \pm 0.002$ & $1.40 \pm 0.03$ & $0.580 \pm 0.033$ & 27.4 & Minho \\
\hline 66B & $43.6 \pm 3.0$ & $35.8 \pm 0.9$ & $12.4 \pm 0.5$ & $53.6 \pm 3.9$ & $11.4 \pm 0.3$ & $0.129 \pm 0.018$ & $0.181 \pm 0.005$ & $1.33 \pm 0.03$ & $0.566 \pm 0.034$ & 27.4 & \\
\hline $67 \mathrm{~A}$ & $58.6 \pm 1.1$ & $32.9 \pm 1.2$ & $11.0 \pm 0.9$ & $52.7 \pm 3.8$ & $12.3 \pm 0.5$ & $0.143 \pm 0.007$ & $0.161 \pm 0.002$ & $1.31 \pm 0.02$ & $0.516 \pm 0.030$ & 23.8 & Minho \\
\hline 67B & $49.3 \pm 2.5$ & $33.4 \pm 1.2$ & $8.7 \pm 0.4$ & $49.0 \pm 1.2$ & $9.9 \pm 0.6$ & $0.125 \pm 0.007$ & $0.194 \pm 0.003$ & $1.36 \pm 0.10$ & $0.499 \pm 0.063$ & 23.8 & \\
\hline 68 & $50.7 \pm 1.2$ & $34.1 \pm 4.4$ & $7.8 \pm 0.6$ & $55.5 \pm 6.1$ & $11.1 \pm 0.5$ & $0.127 \pm 0.015$ & $0.179 \pm 0.003$ & $1.58 \pm 0.10$ & $0.459 \pm 0.063$ & 23.5 & Minho \\
\hline 69 & $70.9 \pm 1.8$ & $44.0 \pm 1.6$ & $12.1 \pm 1.5$ & $56.9 \pm 9.3$ & $11.0 \pm 0.2$ & $0.103 \pm 0.001$ & $0.168 \pm 0.011$ & $1.53 \pm 0.02$ & $0.543 \pm 0.036$ & 27.6 & Oeste \\
\hline 71 & $45.3 \pm 1.3$ & $35.9 \pm 0.7$ & $10.2 \pm 0.8$ & $64.6 \pm 3.7$ & $10.5 \pm 0.4$ & $0.117 \pm 0.014$ & $0.184 \pm 0.003$ & $1.55 \pm 0.05$ & $0.512 \pm 0.017$ & 25.4 & Oeste \\
\hline 72 & $50.9 \pm 0.6$ & $32.5 \pm 2.8$ & $8.1 \pm 0.5$ & $59.0 \pm 4.2$ & $14.1 \pm 2.1$ & $0.152 \pm 0.014$ & $0.178 \pm 0.003$ & $1.59 \pm 0.14$ & $0.486 \pm 0.058$ & 24.0 & Oeste \\
\hline 76 & $69.0 \pm 5.6$ & $41.1 \pm 4.5$ & $10.5 \pm 0.5$ & $71.3 \pm 5.0$ & $10.8 \pm 1.1$ & $0.093 \pm 0.012$ & $0.192 \pm 0.012$ & $1.45 \pm 0.03$ & $0.601 \pm 0.035$ & 25.4 & Algarve \\
\hline 77 & $54.6 \pm 1.8$ & $37.8 \pm 1.4$ & $9.8 \pm 0.3$ & $73.9 \pm 6.5$ & $9.9 \pm 0.2$ & $0.102 \pm 0.021$ & $0.149 \pm 0.001$ & $1.71 \pm 0.10$ & $0.529 \pm 0.020$ & 28.3 & Algarve \\
\hline $78 \mathrm{~A}$ & $59.8 \pm 1.8$ & $36.8 \pm 0.8$ & $10.5 \pm 0.5$ & $71.6 \pm 3.8$ & $10.2 \pm 1.0$ & $0.074 \pm 0.005$ & $0.146 \pm 0.004$ & $1.80 \pm 0.08$ & $0.564 \pm 0.023$ & 29.6 & Algarve \\
\hline 79 & $44.8 \pm 1.1$ & $33.1 \pm 3.6$ & $9.3 \pm 1.4$ & $50.8 \pm 6.8$ & $12.4 \pm 1.3$ & $0.116 \pm 0.026$ & $0.176 \pm 0.003$ & $1.62 \pm 0.14$ & $0.474 \pm 0.033$ & 26.5 & Algarve \\
\hline $80 \mathrm{~A}$ & $58.9 \pm 0.4$ & $36.2 \pm 0.8$ & $6.5 \pm 0.7$ & $67.2 \pm 8.6$ & $14.4 \pm 1.0$ & $0.122 \pm 0.012$ & $0.178 \pm 0.007$ & $1.46 \pm 0.02$ & $0.538 \pm 0.035$ & 27.8 & Algarve \\
\hline $80 \mathrm{~B}$ & $52.6 \pm 2.4$ & $40.6 \pm 5.9$ & $7.7 \pm 1.3$ & $76.1 \pm 3.8$ & $15.2 \pm 1.1$ & $0.123 \pm 0.017$ & $0.163 \pm 0.005$ & $1.75 \pm 0.18$ & $0.564 \pm 0.034$ & 27.8 & \\
\hline 81 & $48.5 \pm 0.5$ & $41.9 \pm 2.1$ & $12.4 \pm 0.7$ & $83.7 \pm 5.0$ & $10.1 \pm 0.6$ & $0.117 \pm 0.016$ & $0.168 \pm 0.002$ & $1.81 \pm 0.04$ & $0.570 \pm 0.017$ & 29.5 & Guarda \\
\hline 82 & $31.2 \pm 2.6$ & $26.7 \pm 3.0$ & $7.7 \pm 1.1$ & $49.2 \pm 5.2$ & $12.0 \pm 1.4$ & $0.123 \pm 0.032$ & $0.208 \pm 0.012$ & $1.33 \pm 0.06$ & $0.458 \pm 0.044$ & 25.7 & Açores \\
\hline 83 & $43.1 \pm 3.5$ & $28.8 \pm 2.1$ & $7.2 \pm 0.9$ & $54.6 \pm 5.2$ & $11.4 \pm 0.9$ & $0.174 \pm 0.014$ & $0.188 \pm 0.009$ & $1.34 \pm 0.02$ & $0.432 \pm 0.036$ & 26.0 & Viseu \\
\hline 85 & $41.4 \pm 0.6$ & $36.3 \pm 2.8$ & $10.1 \pm 0.8$ & $63.2 \pm 9.7$ & $16.1 \pm 1.3$ & $0.090 \pm 0.016$ & $0.192 \pm 0.009$ & $1.60 \pm 0.02$ & $0.535 \pm 0.056$ & 28.1 & Viseu \\
\hline $86 \mathrm{~A}$ & $60.2 \pm 0.6$ & $33.8 \pm 0.7$ & $10.5 \pm 0.2$ & $63.8 \pm 2.1$ & $10.7 \pm 0.8$ & $0.120 \pm 0.010$ & $0.160 \pm 0.004$ & $1.64 \pm 0.03$ & $0.421 \pm 0.019$ & 25.6 & Viseu \\
\hline 86B & $42.3 \pm 2.2$ & $37.7 \pm 2.1$ & $8.7 \pm 0.9$ & $59.9 \pm 3.1$ & $10.1 \pm 0.2$ & $0.102 \pm 0.005$ & $0.179 \pm 0.004$ & $1.78 \pm 0.13$ & $0.438 \pm 0.033$ & & Viseu \\
\hline 87 & $71.7 \pm 1.3$ & $35.7 \pm 2.6$ & $12.4 \pm 1.0$ & $52.7 \pm 1.0$ & $10.2 \pm 0.3$ & $0.124 \pm 0.018$ & $0.148 \pm 0.005$ & $1.76 \pm 0.28$ & $0.496 \pm 0.015$ & 25.5 & Viseu \\
\hline 88 & $64.3 \pm 0.9$ & $34.8 \pm 1.6$ & $11.0 \pm 0.3$ & $61.8 \pm 3.7$ & $11.1 \pm 0.5$ & $0.125 \pm 0.004$ & $0.151 \pm 0.001$ & $1.80 \pm 0.03$ & $0.534 \pm 0.025$ & 26.4 & Viseu \\
\hline $89 \mathrm{~A}$ & $61.1 \pm 2.5$ & $36.0 \pm 1.5$ & $10.5 \pm 1.3$ & $64.5 \pm 5.1$ & $12.5 \pm 1.1$ & $0.125 \pm 0.016$ & $0.177 \pm 0.005$ & $1.36 \pm 0.07$ & $0.480 \pm 0.044$ & 25.4 & Viseu \\
\hline 90 & $38.1 \pm 0.5$ & $43.4 \pm 2.6$ & $10.9 \pm 0.2$ & $88.4 \pm 4.0$ & $12.7 \pm 0.3$ & $0.157 \pm 0.024$ & $0.154 \pm 0.001$ & $1.59 \pm 0.11$ & $0.585 \pm 0.034$ & 27.9 & Lisboa \\
\hline 91 & $86.0 \pm 4.7$ & $27.4 \pm 2.3$ & $5.4 \pm 0.3$ & $41.9 \pm 5.4$ & $15.5 \pm 1.3$ & $0.121 \pm 0.008$ & $0.228 \pm 0.013$ & $2.07 \pm 0.14$ & $0.458 \pm 0.051$ & & Faro \\
\hline $98 \mathrm{~A}$ & $65.0 \pm 3.6$ & $28.4 \pm 1.9$ & $8.7 \pm 1.4$ & $52.3 \pm 4.6$ & $13.3 \pm 0.5$ & $0.086 \pm 0.021$ & $0.192 \pm 0.009$ & $1.75 \pm 0.03$ & $0.558 \pm 0.169$ & 28.9 & Faro \\
\hline $99 \mathrm{~A}$ & $40.0 \pm 0.9$ & $37.1 \pm 3.0$ & $6.5 \pm 0.6$ & $61.7 \pm 4.7$ & $20.0 \pm 2.1$ & $0.137 \pm 0.014$ & $0.193 \pm 0.011$ & $1.76 \pm 0.01$ & $0.498 \pm 0.025$ & & Faro \\
\hline 100 & $35.9 \pm 4.8$ & $37.4 \pm 1.5$ & $10.9 \pm 0.9$ & $67.3 \pm 1.0$ & $13.2 \pm 1.0$ & $0.142 \pm 0.028$ & $0.172 \pm 0.008$ & $1.58 \pm 0.08$ & $0.550 \pm 0.019$ & 27.0 & Tavira \\
\hline 101 & $37.7 \pm 1.5$ & $31.4 \pm 0.4$ & $5.7 \pm 0.5$ & $54.1 \pm 2.1$ & $11.9 \pm 0.4$ & $0.099 \pm 0.006$ & $0.202 \pm 0.005$ & $1.29 \pm 0.03$ & $0.540 \pm 0.128$ & 23.3 & Faro \\
\hline 102 & $53.1 \pm 4.5$ & $29.7 \pm 1.8$ & $4.7 \pm 1.1$ & $49.2 \pm 3.6$ & $15.0 \pm 1.5$ & $0.158 \pm 0.011$ & $0.182 \pm 0.006$ & $1.69 \pm 0.14$ & $0.353 \pm 0.085$ & - & Faro \\
\hline 103 & $48.4 \pm 1.9$ & $36.3 \pm 1.7$ & $12.7 \pm 0.4$ & $68.9 \pm 7.6$ & $11.7 \pm 0.6$ & $0.110 \pm 0.016$ & $0.207 \pm 0.006$ & $1.39 \pm 0.4$ & $0.604 \pm 0.011$ & 28.9 & Loulé \\
\hline 105 & $43.0 \pm 0.5$ & $33.7 \pm 1.8$ & $11.2 \pm 0.2$ & $66.5 \pm 6.6$ & $11.8 \pm 1.1$ & $0.132 \pm 0.007$ & $0.211 \pm 0.014$ & $1.46 \pm 0.08$ & $0.470 \pm 0.019$ & 23.4 & Tavira \\
\hline 106 & $42.7 \pm 1.0$ & $35.3 \pm 0.9$ & $13.3 \pm 0.5$ & $59.3 \pm 3.6$ & $16.5 \pm 0.6$ & $0.174 \pm 0.023$ & $0.204 \pm 0.004$ & $1.65 \pm 0.02$ & $0.537 \pm 0.027$ & 27.0 & Faro \\
\hline 107 & $41.1 \pm 0.4$ & $45.3 \pm 2.7$ & $10.8 \pm 0.3$ & $80.0 \pm 6.9$ & $15.6 \pm 0.5$ & $0.176 \pm 0.020$ & $0.185 \pm 0.006$ & $1.47 \pm 0.07$ & $0.649 \pm 0.029$ & 28.5 & Tavira \\
\hline $108 \mathrm{~A}$ & $33.6 \pm 2.5$ & $34.0 \pm 1.8$ & $10.4 \pm 0.4$ & $52.1 \pm 3.4$ & $15.0 \pm 1.2$ & $0.216 \pm 0.025$ & $0.170 \pm 0.005$ & $1.35 \pm 0.08$ & $0.513 \pm 0.006$ & 23.9 & Tavira \\
\hline $108 \mathrm{C}$ & $42.4 \pm 2.0$ & $33.9 \pm 0.8$ & $12.2 \pm 0.3$ & $60.6 \pm 6.3$ & $11.8 \pm 0.9$ & $0.205 \pm 0.009$ & $0.167 \pm 0.002$ & $1.36 \pm 0.03$ & $0.487 \pm 0.015$ & 24.0 & Loulé \\
\hline 109 & $36.3 \pm 2.3$ & $36.0 \pm 3.3$ & $9.9 \pm 1.0$ & $66.0 \pm 10.0$ & $12.9 \pm 0.6$ & $0.172 \pm 0.014$ & $0.175 \pm 0.001$ & $1.57 \pm 0.08$ & $0.797 \pm 0.402$ & 27.1 & Faro \\
\hline 111B & $28.2 \pm 2.1$ & $43.5 \pm 2.0$ & $10.9 \pm 1.2$ & $63.1 \pm 4.3$ & $11.2 \pm 0.4$ & $0.135 \pm 0.021$ & $0.197 \pm 0.018$ & $1.85 \pm 0.16$ & $0.554 \pm 0.030$ & 26.7 & Faro \\
\hline 112 & $27.0 \pm 1.4$ & $33.7 \pm 1.8$ & $9.0 \pm 1.0$ & $49.3 \pm 1.4$ & $12.9 \pm 0.6$ & $0.111 \pm 0.010$ & $0.187 \pm 0.012$ & $1.52 \pm 0.09$ & $0.475 \pm 0.019$ & 25.5 & Tavira \\
\hline 113 & $42.9 \pm 0.5$ & $36.0 \pm 1.0$ & $10.4 \pm 0.6$ & $62.0 \pm 11.5$ & $10.5 \pm 1.1$ & $0.132 \pm 0.016$ & $0.168 \pm 0.012$ & $1.67 \pm 0.18$ & $0.470 \pm 0.034$ & 21.7 & Tavira \\
\hline 114 & $35.5 \pm 3.0$ & $26.2 \pm 3.5$ & $10.5 \pm 1.0$ & $61.9 \pm 1.4$ & $12.4 \pm 0.1$ & $0.116 \pm 0.012$ & $0.242 \pm 0.009$ & $1.44 \pm 0.07$ & $0.496 \pm 0.034$ & 25.9 & Faro \\
\hline 116 & $35.1 \pm 0.3$ & $31.6 \pm 0.5$ & $10.5 \pm 0.6$ & $55.1 \pm 6.3$ & $10.7 \pm 1.0$ & $0.156 \pm 0.007$ & $0.217 \pm 0.013$ & $1.42 \pm 0.08$ & $0.476 \pm 0.017$ & 23.7 & Loulé \\
\hline 118 & $65.3 \pm 2.2$ & $34.5 \pm 2.6$ & $9.2 \pm 1.1$ & $55.6 \pm 4.4$ & $10.0 \pm 0.8$ & $0.139 \pm 0.002$ & $0.175 \pm 0.005$ & $1.62 \pm 0.05$ & $0.493 \pm 0.042$ & 24.6 & Faro \\
\hline 119 & $32.8 \pm 3.8$ & $41.0 \pm 1.5$ & $10.1 \pm 0.3$ & $56.2 \pm 1.2$ & $11.0 \pm 0.5$ & $0.107 \pm 0.016$ & $0.213 \pm 0.014$ & $2.12 \pm 0.14$ & $0.589 \pm 0.028$ & 25.8 & Tavira \\
\hline 120 & $45.4 \pm 2.2$ & $29.3 \pm 3.0$ & $9.5 \pm 1.0$ & $54.9 \pm 7.1$ & $13.5 \pm 2.1$ & $0.131 \pm 0.015$ & $0.211 \pm 0.019$ & $1.66 \pm 0.10$ & $0.430 \pm 0.039$ & 25.3 & Algarve \\
\hline 121 & $66.9 \pm 2.6$ & $29.1 \pm 2.6$ & $9.6 \pm 1.1$ & $53.6 \pm 9.7$ & $8.9 \pm 0.6$ & $0.109 \pm 0.004$ & $0.192 \pm 0.008$ & $1.70 \pm 0.03$ & $0.543 \pm 0.071$ & 26.0 & Algarve \\
\hline 125 & $42.7 \pm 3.0$ & $35.1 \pm 2.7$ & $8.2 \pm 1.0$ & $60.3 \pm 9.2$ & $11.8 \pm 0.6$ & $0.171 \pm 0.018$ & $0.187 \pm 0.016$ & $1.52 \pm 0.14$ & $0.480 \pm 0.054$ & & Algarve \\
\hline 126 & $46.3 \pm 1.7$ & $30.8 \pm 1.9$ & $8.7 \pm 0.3$ & $54.1 \pm 10.3$ & $17.8 \pm 2.7$ & $0.165 \pm 0.028$ & $0.175 \pm 0.007$ & $1.55 \pm 0.09$ & $0.472 \pm 0.040$ & & Faro \\
\hline 127 & $56.2 \pm 2.5$ & $34.8 \pm 1.5$ & $8.8 \pm 1.0$ & $43.9 \pm 1.6$ & $14.9 \pm 2.1$ & $0.093 \pm 0.012$ & $0.165 \pm 0.008$ & $1.62 \pm 0.11$ & $0.439 \pm 0.050$ & 25.4 & Faro \\
\hline 129 & $40.4 \pm 1.2$ & $26.8 \pm 2.5$ & $8.4 \pm 0.4$ & $39.5 \pm 3.6$ & $15.0 \pm 0.1$ & $0.247 \pm 0.060$ & $0.206 \pm 0.012$ & $1.77 \pm 0.12$ & $0.432 \pm 0.027$ & 26.4 & Faro \\
\hline 131 & $41.3 \pm 0.7$ & $38.9 \pm 4.9$ & $8.6 \pm 1.0$ & $57.1 \pm 11.8$ & $18.6 \pm 7.2$ & $0.136 \pm 0.015$ & $0.200 \pm 0.001$ & $1.71 \pm 0.05$ & $0.528 \pm 0.032$ & - & Faro \\
\hline 132 & $63.8 \pm 0.8$ & $33.7 \pm 1.2$ & $10.2 \pm 0.4$ & $58.2 \pm 3.7$ & $10.7 \pm 0.4$ & $0.104 \pm 0.001$ & $0.179 \pm 0.014$ & $1.69 \pm 0.09$ & $0.540 \pm 0.005$ & 27.0 & Faro \\
\hline
\end{tabular}




\begin{tabular}{|c|c|c|c|c|c|c|c|c|c|c|c|}
\hline 134 & $79.0 \pm 0.3$ & $23.8 \pm 2.5$ & $8.8 \pm 0.3$ & $54.2 \pm 4.7$ & $15.7 \pm 1.5$ & $0.174 \pm 0.004$ & $0.173 \pm 0.002$ & $1.66 \pm 0.06$ & $0.524 \pm 0.035$ & & Faro \\
\hline 136 & $75.1 \pm 2.2$ & $23.9 \pm 1.9$ & $8.2 \pm 0.7$ & $34.0 \pm 4.1$ & $11.4 \pm 0.8$ & $0.143 \pm 0.009$ & $0.173 \pm 0.009$ & $1.54 \pm 0.04$ & $0.478 \pm 0.044$ & & Faro \\
\hline 146 & $53.0 \pm 1.9$ & $41.1 \pm 2.1$ & $7.9 \pm 0.8$ & $50.4 \pm 1.7$ & $17.2 \pm 2.3$ & $0.186 \pm 0.023$ & $0.202 \pm 0.003$ & $1.34 \pm 0.09$ & $0.441 \pm 0.067$ & & Faro \\
\hline 147 & $47.2 \pm 1.1$ & $30.0 \pm 0.8$ & $9.8 \pm 1.0$ & $44.6 \pm 1.9$ & $17.4 \pm 1.6$ & $0.153 \pm 0.020$ & $0.195 \pm 0.005$ & $1.72 \pm 0.10$ & $0.604 \pm 0.021$ & & Faro \\
\hline 151 & $80.0 \pm 5.7$ & $29.4 \pm 5.8$ & $7.2 \pm 0.8$ & $47.0 \pm 14.6$ & $17.5 \pm 8.6$ & $0.122 \pm 0.013$ & $0.205 \pm 0.021$ & $1.89 \pm 0.13$ & $0.535 \pm 0.025$ & & Faro \\
\hline 155 & $53.0 \pm 1.4$ & $35.9 \pm 2.3$ & $10.7 \pm 1.3$ & $55.3 \pm 7.3$ & $16.9 \pm 2.1$ & $0.116 \pm 0.012$ & $0.209 \pm 0.009$ & $1.71 \pm 0.05$ & $0.418 \pm 0.038$ & 26.0 & Faro \\
\hline 156 & $63.9 \pm 1.4$ & $32.7 \pm 3.0$ & $8.9 \pm 1.0$ & $55.5 \pm 8.0$ & $11.1 \pm 0.6$ & $0.093 \pm 0.013$ & $0.207 \pm 0.010$ & $1.80 \pm 0.05$ & $0.389 \pm 0.030$ & 26.4 & Faro \\
\hline 157 & $46.0 \pm 1.5$ & $34.8 \pm 1.6$ & $10.3 \pm 0.7$ & $57.6 \pm 4.6$ & $16.2 \pm 1.6$ & $0.141 \pm 0.009$ & $0.200 \pm 0.008$ & $1.59 \pm 0.07$ & $0.412 \pm 0.027$ & 25.5 & Faro \\
\hline 158 & $50.0 \pm 1.4$ & $31.4 \pm 1.8$ & $11.5 \pm 0.1$ & $42.9 \pm 2.1$ & $12.5 \pm 1.7$ & $0.145 \pm 0.026$ & $0.215 \pm 0.007$ & $1.76 \pm 0.09$ & $0.493 \pm 0.203$ & 29.7 & Faro \\
\hline 161 & $54.6 \pm 1.8$ & $38.0 \pm 3.9$ & $8.5 \pm 1.0$ & $72.7 \pm 1.2$ & $16.5 \pm 2.4$ & $0.168 \pm 0.027$ & $0.227 \pm 0.002$ & $1.52 \pm 0.05$ & $0.476 \pm 0.088$ & 26.2 & Oeste \\
\hline 167 & $49.3 \pm 1.8$ & $31.1 \pm 2.9$ & $10.2 \pm 0.9$ & $43.1 \pm 3.4$ & $13.2 \pm 1.4$ & $0.167 \pm 0.009$ & $0.185 \pm 0.008$ & $1.69 \pm 0.07$ & $0.428 \pm 0.032$ & 28.4 & Oeste \\
\hline 168 & $43.2 \pm 2.4$ & $33.0 \pm 2.5$ & $7.5 \pm 1.1$ & $51.7 \pm 8.9$ & $10.2 \pm 1.2$ & $0.114 \pm 0.007$ & $0.199 \pm 0.015$ & $1.71 \pm 0.07$ & $0.458 \pm 0.020$ & 29.4 & Oeste \\
\hline 174 & $66.4 \pm 3.2$ & $36.0 \pm 0.8$ & $11.2 \pm 1.8$ & $47.2 \pm 2.5$ & $10.8 \pm 0.2$ & $0.119 \pm 0.016$ & $0.182 \pm 0.009$ & $1.52 \pm 0.03$ & $0.496 \pm 0.055$ & 26.9 & Oeste \\
\hline 175 & $63.6 \pm 2.1$ & $33.2 \pm 4.5$ & $10.7 \pm 1.6$ & $40.1 \pm 4.6$ & $11.2 \pm 0.7$ & $0.144 \pm 0.026$ & $0.174 \pm 0.006$ & $1.69 \pm 0.06$ & $0.467 \pm 0.038$ & & Oeste \\
\hline 199 & $48.9 \pm 2.2$ & $28.4 \pm 3.4$ & $12.6 \pm 0.8$ & $56.7 \pm 4.1$ & $11.3 \pm 1.0$ & $0.106 \pm 0.012$ & $0.186 \pm 0.006$ & $1.63 \pm 0.06$ & $0.472 \pm 0.029$ & 22.6 & Oeste \\
\hline 205 & $58.6 \pm 5.5$ & $33.6 \pm 1.1$ & $6.8 \pm 0.2$ & $56.2 \pm 2.4$ & $11.4 \pm 0.5$ & $0.099 \pm 0.003$ & $0.192 \pm 0.008$ & $1.64 \pm 0.13$ & $0.454 \pm 0.048$ & 24.1 & Oeste \\
\hline 220 & $55.4 \pm 1.4$ & $19.7 \pm 2.7$ & $6.4 \pm 0.3$ & $52.0 \pm 3.5$ & $9.7 \pm 0.6$ & $0.068 \pm 0.013$ & $0.178 \pm 0.007$ & $1.46 \pm 0.06$ & $0.502 \pm 0.047$ & 28.0 & Oeste \\
\hline 222 & $32.3 \pm 2.2$ & $36.7 \pm 0.3$ & $10.9 \pm 0.9$ & $85.5 \pm 2.9$ & $18.8 \pm 1.2$ & $0.293 \pm 0.040$ & $0.212 \pm 0.010$ & $1.34 \pm 0.10$ & $0.383 \pm 0.021$ & 21.1 & Oeste \\
\hline 224 & $56.6 \pm 0.4$ & $37.2 \pm 2.3$ & $8.9 \pm 1.1$ & $65.5 \pm 4.6$ & $12.5 \pm 0.6$ & $0.153 \pm 0.004$ & $0.184 \pm 0.008$ & $1.57 \pm 0.16$ & $0.552 \pm 0.019$ & 29.2 & Bragança \\
\hline 226 & $49.8 \pm 1.6$ & $28.0 \pm 0.9$ & $9.3 \pm 0.5$ & $38.8 \pm 3.8$ & $13.4 \pm 0.5$ & $0.141 \pm 0.022$ & $0.178 \pm 0.001$ & $1.39 \pm 0.09$ & $0.410 \pm 0.010$ & 24.0 & Bragança \\
\hline 229 & $46.5 \pm 2.0$ & $35.0 \pm 2.1$ & $11.9 \pm 0.7$ & $83.5 \pm 8.1$ & $16.7 \pm 0.8$ & $0.174 \pm 0.021$ & $0.228 \pm 0.028$ & $1.24 \pm 0.67$ & $0.478 \pm 0.093$ & 26.2 & Bragança \\
\hline 233 & $63.3 \pm 4.4$ & $11.5 \pm 1.1$ & $5.1 \pm 0.6$ & $32.2 \pm 0.8$ & $8.0 \pm 0.3$ & $0.126 \pm 0.014$ & $0.199 \pm 0.010$ & $1.43 \pm 0.03$ & $0.613 \pm 0.023$ & 22.6 & Bragança \\
\hline 234 & $55.8 \pm 2.5$ & $20.3 \pm 2.5$ & $9.3 \pm 1.6$ & $66.5 \pm 1.8$ & $13.3 \pm 1.6$ & $0.123 \pm 0.010$ & $0.192 \pm 0.014$ & $1.52 \pm 0.09$ & $0.541 \pm 0.019$ & 22.2 & Bragança \\
\hline 243 & $45.6 \pm 1.7$ & $16.1 \pm 2.4$ & $10.3 \pm 1.6$ & $41.9 \pm 4.6$ & $15.3 \pm 2.0$ & $0.142 \pm 0.045$ & $0.217 \pm 0.011$ & $1.35 \pm 0.15$ & $0.564 \pm 0.039$ & 27.2 & Bragança \\
\hline 245 & $60.8 \pm 0.8$ & $32.9 \pm 2.9$ & $9.3 \pm 0.3$ & $60.6 \pm 2.2$ & $11.0 \pm 0.8$ & $0.125 \pm 0.015$ & $0.193 \pm 0.010$ & $1.41 \pm 0.09$ & $0.438 \pm 0.028$ & 24.6 & Bragança \\
\hline 246 & $70.6 \pm 6.0$ & $35.8 \pm 4.9$ & $10.5 \pm 1.3$ & $73.9 \pm 3.0$ & $15.2 \pm 2.0$ & $0.196 \pm 0.006$ & $0.179 \pm 0.027$ & $1.56 \pm 0.16$ & $0.487 \pm 0.114$ & 24.6 & Bragança \\
\hline 248 & $45.0 \pm 0.5$ & $37.5 \pm 2.4$ & $9.9 \pm 1.1$ & $61.5 \pm 8.1$ & $13.3 \pm 2.3$ & $0.154 \pm 0.026$ & $0.184 \pm 0.002$ & $1.49 \pm 0.13$ & $0.493 \pm 0.008$ & 26.6 & Miranda do Douro \\
\hline 249 & $59.5 \pm 2.1$ & $34.2 \pm 3.1$ & $9.7 \pm 1.1$ & $85.8 \pm 8.1$ & $12.7 \pm 2.1$ & $0.087 \pm 0.028$ & $0.198 \pm 0.004$ & $1.49 \pm 0.05$ & $0.507 \pm 0.060$ & 26.0 & Miranda do Douro \\
\hline 250 & $46.8 \pm 2.2$ & $34.7 \pm 2.0$ & $9.3 \pm 0.7$ & $60.2 \pm 9.3$ & $15.6 \pm 1.0$ & $0.162 \pm 0.019$ & $0.165 \pm 0.013$ & $1.45 \pm 0.08$ & $0.462 \pm 0.026$ & 26.1 & Miranda do Douro \\
\hline 257 & $36.2 \pm 1.4$ & $36.3 \pm 1.4$ & $9.0 \pm 0.1$ & $49.6 \pm 3.4$ & $16.3 \pm 0.9$ & $0.162 \pm 0.007$ & $0.222 \pm 0.019$ & $1.59 \pm 0.03$ & $0.528 \pm 0.024$ & 26.2 & Algarve \\
\hline 262 & $23.9 \pm 1.4$ & $35.3 \pm 2.3$ & $8.2 \pm 1.3$ & $66.0 \pm 8.2$ & $19.5 \pm 1.0$ & $0.152 \pm 0.004$ & $0.208 \pm 0.006$ & $1.57 \pm 0.14$ & $0.479 \pm 0.038$ & & Cabo Verde \\
\hline $275 \mathrm{~A}$ & $100.4 \pm 3.1$ & $29.8 \pm 1.2$ & $8.5 \pm 0.7$ & $48.4 \pm 1.3$ & $10.2 \pm 1.4$ & $0.076 \pm 0.002$ & $0.198 \pm 0.005$ & $1.56 \pm 0.06$ & $0.339 \pm 0.021$ & & Barcelos \\
\hline 275B & $97.3 \pm 4.0$ & $27.8 \pm 1.7$ & $7.0 \pm 0.3$ & $41.4 \pm 4.7$ & $9.8 \pm 1.3$ & $0.120 \pm 0.023$ & $0.183 \pm 0.020$ & $1.59 \pm 0.01$ & $0.332 \pm 0.030$ & & Barcelos \\
\hline $275 R$ & $57.8 \pm 0.9$ & $28.9 \pm 0.7$ & $8.8 \pm 1.1$ & $46.0 \pm 5.3$ & $10.7 \pm 0.2$ & $0.127 \pm 0.010$ & $0.169 \pm 0.006$ & $1.54 \pm 0.04$ & $0.405 \pm 0.010$ & & Barcelos \\
\hline 276 & $34.4 \pm 1.0$ & $30.0 \pm 1.5$ & $8.2 \pm 0.4$ & $43.7 \pm 5.6$ & $10.6 \pm 0.2$ & $0.138 \pm 0.003$ & $0.197 \pm 0.006$ & $1.45 \pm 0.09$ & $0.426 \pm 0.020$ & 23.2 & Barcelos \\
\hline 277 & $35.5 \pm 1.4$ & $31.0 \pm 0.3$ & $10.0 \pm 0.3$ & $53.3 \pm 9.4$ & $12.2 \pm 1.1$ & $0.136 \pm 0.013$ & $0.128 \pm 0.010$ & $1.70 \pm 0.04$ & $0.483 \pm 0.012$ & 22.9 & Barcelos \\
\hline 278 & $36.3 \pm 1.6$ & $35.2 \pm 0.8$ & $9.2 \pm 0.6$ & $61.8 \pm 3.5$ & $16.1 \pm 2.2$ & $0.154 \pm 0.029$ & $0.224 \pm 0.015$ & $1.71 \pm 0.07$ & $0.507 \pm 0.137$ & 21.9 & Barcelos \\
\hline 281 & $63.9 \pm 4.2$ & $32.9 \pm 2.3$ & $10.3 \pm 0.5$ & $60.2 \pm 2.4$ & $17.3 \pm 0.6$ & $0.189 \pm 0.007$ & $0.198 \pm 0.014$ & $1.65 \pm 0.04$ & $0.393 \pm 0.149$ & 22.3 & Barcelos \\
\hline 283 & $56.5 \pm 1.0$ & $33.1 \pm 1.6$ & $10.8 \pm 1.0$ & $48.4 \pm 5.8$ & $12.5 \pm 1.0$ & $0.145 \pm 0.030$ & $0.198 \pm 0.009$ & $1.74 \pm 0.09$ & $0.537 \pm 0.155$ & & Barcelos \\
\hline 285 & $44.9 \pm 2.2$ & $31.1 \pm 1.3$ & $8.0 \pm 1.4$ & $50.1 \pm 6.1$ & $14.3 \pm 1.4$ & $0.172 \pm 0.028$ & $0.197 \pm 0.009$ & $1.57 \pm 0.12$ & $0.411 \pm 0.046$ & 26.5 & Barcelos \\
\hline 291 & $61.3 \pm 2.7$ & $42.6 \pm 1.7$ & $8.6 \pm 1.6$ & $63.3 \pm 5.6$ & $20.1 \pm 1.1$ & $0.174 \pm 0.028$ & $0.187 \pm 0.009$ & $1.66 \pm 0.11$ & $0.491 \pm 0.057$ & & Barcelos \\
\hline $291 \mathrm{E}$ & $54.5 \pm 2.0$ & $30.5 \pm 2.3$ & $9.8 \pm 1.0$ & $41.3 \pm 6.4$ & $16.3 \pm 1.3$ & $0.099 \pm 0.004$ & $0.204 \pm 0.013$ & $1.77 \pm 0.04$ & $0.557 \pm 0.067$ & & Barcelos \\
\hline 293 & $40.5 \pm 2.4$ & $34.4 \pm 0.9$ & $10.3 \pm 1.0$ & $51.6 \pm 6.1$ & $13.0 \pm 0.7$ & $0.125 \pm 0.036$ & $0.198 \pm 0.009$ & $1.85 \pm 0.10$ & $0.457 \pm 0.028$ & & Barcelos \\
\hline 297 & $24.0 \pm 0.4$ & $34.0 \pm 1.9$ & $11.6 \pm 0.7$ & $61.1 \pm 6.1$ & $18.5 \pm 1.3$ & $0.165 \pm 0.014$ & $0.239 \pm 0.008$ & $1.81 \pm 0.13$ & $0.503 \pm 0.026$ & 22.5 & Barcelos \\
\hline 298 & $24.8 \pm 0.1$ & $34.2 \pm 1.2$ & $10.7 \pm 0.2$ & $62.7 \pm 3.5$ & $15.9 \pm 0.2$ & $0.137 \pm 0.001$ & $0.247 \pm 0.010$ & $1.57 \pm 0.02$ & $0.489 \pm 0.025$ & & Barcelos \\
\hline Ratio percentile & 3.0 & 2.2 & 3.8 & 2.3 & 2.2 & 2.700 & 1.600 & 1.5 & 1.700 & 1.4 & \\
\hline $\operatorname{Max}$ & 100.4 & 45.3 & 13.5 & 88.4 & 20.1 & 0.293 & 0.253 & 2.12 & 0.797 & 30.0 & \\
\hline Min & 3.0 & 11.5 & 4.7 & 32.2 & 8.0 & 0.068 & 0.128 & 1.24 & 0.497 & 21.1 & \\
\hline
\end{tabular}

a Protein content as determined by Palha et al. (1988) in 124 accessions.

b Ratio percentile $97.5 \% / 2.5 \%$. 


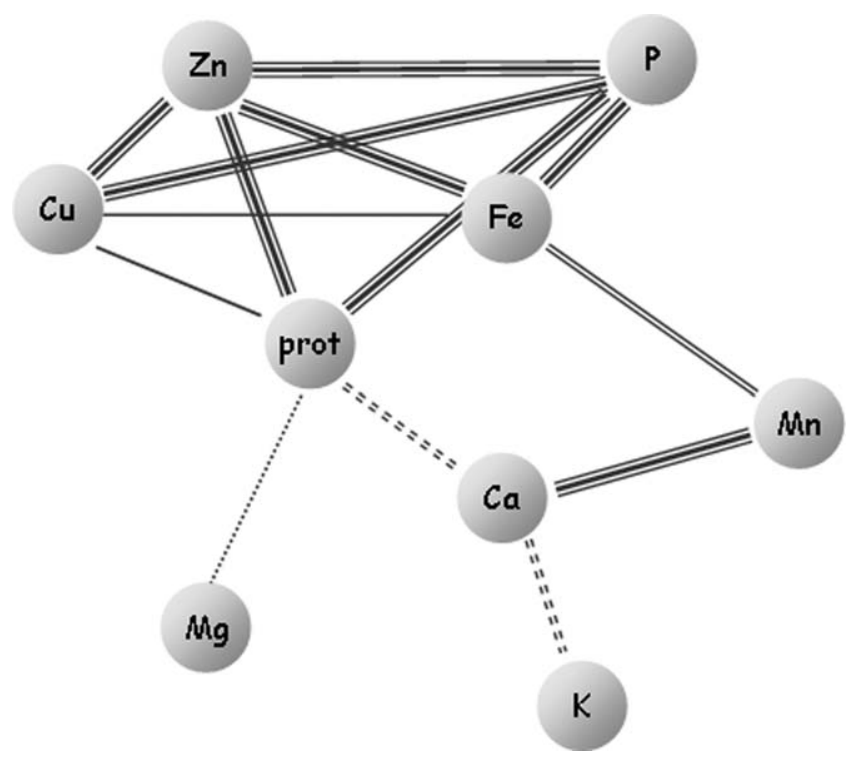

Fig. 2. Graphical representation of the correlations between the several elements analysed in the $P$. vulgaris accessions $(n=155)$, which results from the total correlation matrix: $P \leq 0.0001$ (triple line), $P \leq 0.001$ (double line) and $P \leq 0.05$ (single line). Negative correlations are similarly illustrated by dotted lines.

analysed it was possible to detect many accessions with very high seed concentration of that mineral. Particularly high levels of Fe, $\mathrm{Zn}, \mathrm{P}$ and Ca were observed, but several accessions with low levels of seed minerals were also identified. For the micronutrient concentrations, accessions varied from 2.5-fold for Mn to 3.9-fold for $\mathrm{Zn}$, while for the macronutrient concentrations, accessions varied from 1.6-fold for $\mathrm{P}$ to 4.3 -fold for Ca. Taking into consideration the coefficient of variation, for micronutrients the observed variation ranged from $15.3 \%$ for $\mathrm{Zn}$ to $20.6 \%$ for $\mathrm{Mn}$, and for macronutrients from $10.0 \%$ for $\mathrm{K}$ to $25.5 \%$ for Ca. These results indicate the existence in the germplasm collection of a significant degree of genetic variability that seems particularly relevant for $\mathrm{Cu}, \mathrm{Ca}, \mathrm{Fe}, \mathrm{Zn}$ and $\mathrm{Mn}$.

A total correlation matrix analysis (Fig. 2) revealed two strong sets of correlations, one associating protein, $\mathrm{Zn}, \mathrm{Fe}, \mathrm{P}$ and $\mathrm{Cu}$ $(P \leq 0.0001)$, and the other $\mathrm{Ca}$ and $\mathrm{Mn}(P \leq 0.0001)$. When partial correlations were considered, the very strong associations $\mathrm{Zn}-\mathrm{Fe}$, $\mathrm{Zn}-\mathrm{Cu}, \mathrm{Cu}-\mathrm{P}$ and $\mathrm{Ca}-\mathrm{Mn}$ were confirmed and it was possible to classify the Fe-P association as a strong correlation "forced" by the other very strong ones. We could not find any correlation between mineral composition and the geographical origin of the accessions, which might be due to the high soil heterogeneity in Portugal. We could not find any correlation between mineral composition and seed size either.

A PCA showed that $\mathrm{Zn}$, Fe and $\mathrm{Cu}$ are highly correlated to the first component (27\% of the variability), Ca and $\mathrm{Mn}$ to the second component (22\% of the variability) and $\mathrm{Mg}$ and $\mathrm{K}$ to the third component (15\% of the variability) (Fig. 3 ).

\section{Discussion}

Considering the great value of traditional plant germplasm collections, it is important to characterise them with respect to their nutritional value. Studies on Portuguese grain legume germplasm (Palha et al., 1988; Pereira and Tavares-de-Sousa, 1996; Pereira et al., 1998, 2006; Rodiño et al., 2001, 2003; Vaz et al., 2004) have focused on growth habits, physiological traits and seed protein content. We have now analysed the seed mineral content of an important Portuguese germplasm collection of common bean (P. vulgaris). The high variability in Fe and $\mathrm{Zn}$ concentration found in Mesoamerican and Andean landraces (Beebe et al., 2000; Moraghan and Grafton, 2001; Moraghan et al., 2002) is also observed in the Portuguese collection, which, additionally, displays high variability in relation to $\mathrm{P}, \mathrm{Mn}, \mathrm{Ca}$ and $\mathrm{Cu}$. This information is potentially important for breeding programs since some accessions have high values of $\mathrm{P}, \mathrm{Zn}, \mathrm{Fe}, \mathrm{Cu}$ and protein. It is also relevant that besides the $\mathrm{Fe}-\mathrm{Zn}$ positive correlation previously reported (Welch et al., 2000) we find strong positive correlations of $\mathrm{P}-\mathrm{Cu}, \mathrm{P}-$ protein and $\mathrm{Ca}-\mathrm{Mn}$.

Despite the detection of these correlations, little information exists on the biochemical processes that underlie them. Concerning the P-proteins correlation we might speculate that it could reflect some kind of association existing in the protein bodies, where protein and phosphorus (as phytate) are accumulated. The $\mathrm{Ca}-\mathrm{Mn}$ correlation highlights the problems associated with $\mathrm{Mn}$ metabolism in grain legumes. Considerable variability can occur in the Mn concentration of seeds, influencing plant growth and development, crop yield and seed quality (Longnecker and Uren, 1990). Manganese toxicity is a major constraint for the production of common bean in tropical and subtropical soils (Gonzalez and Lynch, 1999), but it can be avoided if the soil Ca/Mn ratio is higher than 80 (Bekker et al., 1994). Ca may alleviate Mn phytotoxicity

Fig. 3. Principal component analysis of the several elements ( $\mathrm{Zn}, \mathrm{Cu}, \mathrm{Fe}, \mathrm{Mn}, \mathrm{Ca}, \mathrm{Mg}, \mathrm{P}$ and $\mathrm{K})$ analysed in the $\mathrm{P}$. vulgaris accessions ( $n=155$ ). 
through the inhibition of Mn absorption (Bekker et al., 1994) and translocation to the shoots (Alam et al., 2006). In our study, Mn toxicity during common bean development was not expected because the soil $\mathrm{Ca} / \mathrm{Mn}$ ratio was $\sim 3000$. Interestingly, $\mathrm{Mn}$ accumulation in seeds does not seem to be negatively affected by $\mathrm{Ca}$. On the contrary, we observed a strong positive $\mathrm{Ca}-\mathrm{Mn}$ correlation that was similarly found in Arabidopsis seeds (Vreugdenhil et al., 2004).

When considering the nutrition potentialities of this common bean collection we should emphasise the importance of legumes (in particular common beans) for direct human consumption worldwide (Broughton et al., 2003) and the relevance of grain legumes as mineral suppliers (Welch et al., 2000). Deficiencies in essential mineral cations affect large populations in several parts of the world, as it is well known for Fe and $\mathrm{Zn}$. The importance for the human nutrition of $\mathrm{P}, \mathrm{Cu}, \mathrm{Ca}$ and $\mathrm{Mn}$, in addition to $\mathrm{Zn}$ and $\mathrm{Fe}$ should also be taken into consideration (Solomons and Ruz, 1998). For instance, Mn deficiency has been detected in animals, and it was also observed that high dietary intake of $\mathrm{Ca}, \mathrm{P}$ and Fe reduces Mn absorption (Hathcock, 2004). So, the relevance of the observed $\mathrm{Ca}-\mathrm{Mn}$ strong positive correlation in the common beans should be evaluated in terms of nutritional Mn bioavailability.

Our results, besides expressing the importance of the Portuguese common bean germplasm collection, raise several questions of a physiological and biochemical nature, indicating that processes that culminate in the mineral storage in the seeds are poorly understood. Additional studies are needed to understand the integration of all those processes and their implications regarding animal and human nutrition.

\section{Acknowledgements}

Thanks are due to Eng. Ma Conceição Gonçalves and Eng. José Casimiro Martins (Departamento de Solos da Estação Agronómica Nacional) for helpful discussions and to Dr. Pedro Duarte (Instituto de Tecnologia Nuclear) for help with map drawing. CP acknowledges a grant SFRH/BPD/14535/2003 from Fundação para a Ciência e Tecnologia.

\section{References}

Alam, S., Kodama, R., Akiha, F., Kamei, S., Kawai, S., 2006. Alleviation of manganese phytotoxicity in barley with calcium. Journal of Plant Nutrition 29, 59-74.

Anon., 1971 Analytical methods for atomic adsorption. Agriculture analysis of plant tissue dry ashing procedure. Perkin-Elmer Corporation, Norwalk, Connecticut, USA, pp. AY-4.

Beebe, S., Gonzalez, A.V., Rengifo, J., 2000. Research on trace minerals in the common bean. Food and Nutrition Bulletin 21, 387-391.

Bekker, A.W., Hue, N.V., Yapa, L.G.G., Chase, R.G., 1994. Peanut growth as affected by liming, $\mathrm{Ca}-\mathrm{Mn}$ interactions, and $\mathrm{Cu}$ plus $\mathrm{Zn}$ application to Samoan soils. Plant and Soil 164, 203-211.
Black, C.A., Evans, D.D., White, J.L., Ensminger, L.E., Clark, F.E., 1965. Methods of Soil Analysis: Part 2-Microbiological and Biochemical Properties. American Society of Agronomy, Madison, Wisconsin, USA

Broughton, W.J., Hernández, G., Blair, M., Beebe, S., Gepts, P., Vanderleyden, J., 2003. Beans (Phaseolus spp.) - model food legumes. Plant and Soil 252, 55-128.

Chapman, H.D., Pratt, P.F., 1961. Methods of analysis for soils, plants and waters. University of California, Division of Agricultural Sciences, p. 299

Egner, H., Riehm, H., Domingo, W.R., 1960. Untersuchungen ueber die chemische Bodenanalyse als Grundlage fuer die Beurteilung des Nahrstoffzustandes der Boeden. II. Chemische Extractionsmethoden zur Phosphor- und Kaliumbestinimung. K. Lantbr. Hogsk. Annlr 26, 199-215.

Gonzalez, A., Lynch, J., 1999. Tolerance of tropical common bean genotypes to manganese toxicity: performance under different growing conditions. Journal of Plant Nutrition 22, 511-525.

Hathcock, J.N., 2004. Vitamin and Mineral Safety, 2nd Edition. CRN.

Lindsay, W.L., Norvell, W.A., 1978. Development of a DTPA soil test for zinc, iron, manganese, and copper. Soil Science Society of America Journal 42, 421428 .

Longnecker, N.E., Uren, C., 1990. Factors influencing variability in manganese content of seeds, with emphasison barley (Hordeum vulgare) and white lupins (Lupinus albus). Australian Journal of Agricultural Research 41, 29-37.

Moraghan, J.T., Grafton, K., 2001. Genetic diversity and mineral composition of common bean seed. Journal of the Science of Food and Agriculture 81, 404-408.

Moraghan, J.T., Padilla, J., Etchevers, J.D., Grafton, K., Acosta-Gallegos, J.A., 2002. Iron accumulation in seed of common bean. Plant and Soil 246, 175-183.

Palha, M.G.S., Ricardo, C.P.P., Baeta, J.M.P., 1988. Teor proteico da semente de linhas nacionais de feijoeiro. Agronomia Lusitana 43, 115-131.

Pereira, G., Tavares-de-Sousa, M., 1996. Caracterização morfológica de populações portuguesas de faveira (V. faba L.). Pastagens e Forragens 17, 105-111.

Pereira, G., Mihov, M., Atanassova, D., Costa, R., Stoilova, T., Tavares-de-Sousa, M., 2006. Study of plant variability in a pea collection. Melhoramento 40, 38-46.

Pereira, G., Tavares-de-Sousa, M., Bravo-Lima, M., 1998. Morphological characterization of Portuguese populations of chickpea (Cicer arietinum L.). In: Proceedings of 3rd European Conference on Grain Legumes: Opportunities for High Quality, Healthy and Added-Value Crops to meet European demands, November 1998, Valladolid, Spain, p. 202.

Rodiño, A.P., Santalla, M., De Ron, A.M., Singh, S.P., 2003. A core collection of common bean from the Iberian Peninsula. Euphytica 131, 165-175.

Rodiño, A.P., Santalla, M., Montero, I., Casquero, P.A., De Ron, A.M., 2001. Diversity of common bean (Phaseolus vulgaris L.) germplasm from Portugal. Genetic Resources and Crop Evolution 48, 409-417.

Santalla, M., Rodiño, A.P., De Ron, A.M., 2002. Alloenzyme evidence supporting southwestern Europe as a secondary center of genetic diversity for common bean. Theoretical and Applied Genetics 104, 934-944.

Soil Conservation Service, 1972. Soil survey laboratory methods and procedures for collecting soil samples. Soil Survey Investigations Report $n^{\circ} 1$. Soil Conservation Service, US Department of Agriculture, Washington, D.C.

Solomons, N.W., Ruz, M., 1998. Trace elements requirements in humans: an update. The Journal of Trace Elements in Experimental Medicine 11, 177-195.

Vaz, A.C., Pinheiro, C., Martins, J.M.N., Ricardo, C.P.P., 2004. Cultivar discrimination of Portuguese Lupinus albus by seed protein electrophoresis: the importance of considering "glutelins" and glycoproteins. Field Crops Research 87, 23-34.

Vreugdenhil, D., Aarts, M.G.M., Koornneef, M., Nelissen, H., Ernst, W.H.O., 2004. Natural variation and QTL analysis for cationic mineral content in seeds of Arabidopsis thaliana. Plant, Cell and Environment 27, 828-839.

Wang, T.L., Domoney, C., Hedley, C.L., Casey, R., Grusak, M.A., 2003. Can we improve the nutritional quality of legume seeds? Plant Physiology 131, 886-891.

Welch, R.M., House, W.A., Beebe, S., Cheng, Z., 2000. Genetic selection for enhanced bioavailable levels of iron in bean (Phaseolus vulgaris L.) seeds. Journal of the Science of Food and Agriculture 48, 3576-3580.

WRB, 2006. World reference base for soil resources. A framework for international classification, correlation and communication. World Soil Reports (FAO), p. 103. 